\title{
Primary health care and general practice nurses: What is the nexus?
}

\author{
Authors \\ Elizabeth Patterson \\ $\mathrm{RN}, \mathrm{PhD}$ \\ Centre for Clinical Practice Innovation \\ Griffith University - Gold Coast \\ PMB 50 \\ GCMC 9726
}

Kay Price

$\mathrm{RN}, \mathrm{PhD}$

School of Nursing and Midwifery

University of South Australia

Desley Hegney

RN, PhD

Centre for Rural and Remote Area Health University of Southern Queensland, University of Queensland and Queensland Health 


\title{
Primary health care and general practice nurses: What is the nexus?
}

\begin{abstract}
This paper presents the findings from three separate qualitative studies that sought to explore the current and potential role of nurses employed in general medical practices in Australia. General practitioners', practice nurses' and consumers' views and perceptions were gathered from individual and group interviews. The data indicate that practice nurses (PNs) are involved in first level or primary care of individuals and engage in some form of preventive health care. Some PNs have a family/community focus in addition to their focus of care on individuals. Engagement in health promotion was found to be opportunistic rather than planned and focussed on interventions to free individuals from medically defined diseases, the aim being compliance with therapeutic procedures and advice. The broader concept of health promotion, as documented in the Ottawa Charter for Health Promotion was not pronounced in the PNs' reported practice. Consumers do not articulate confidence in PNs acting autonomously as primary health care practitioners but rather as complementary to general practitioners (GPs), undertaking initial assessment for triage purposes and providing ongoing management, education and support under the GPs' delegation. They would also like them to be family oriented and holistic in their practice; supporting emotional and social needs in the context of their family lives.
\end{abstract}

Key words: general practice, practice nurse, primary health care, qualitative research 
Primary health care and general practice nurses: What is the nexus?

\section{Introduction}

In 1978, Primary Health Care (PHC) was formally recognised in the Declaration of Alma-Ata as the key to achieving the World Health Organisation's goal of 'Health For All by the Year 2000' (WHO, 1978). PHC was seen as the solution to the inadequate illness management systems that had developed throughout the world. It was hoped that PHC would address some of the major inequalities in health observed both within and between countries by a balanced system of treatment and disease prevention. The World Health Organisation (WHO) envisaged that PHC would take place as close as possible to where people live and work and be the first element of a continuing health care process. Additionally, health service collaboration and multiprofessional partnerships were expected to replace professional boundaries and competition.

As a Member State of the WHO, Australia made a commitment to PHC in the signing of the Declaration of Alma-Ata in 1978 and in the formal adoption of the global strategy of Health for All (HFA) in 1981. One of the initiatives implemented to respond to Australia's commitment to PHC was the establishment of the National Health Strategy in 1990. One of the issues paper (National Health Strategy, 1992) emanating from the 'Strategy' identified the vital role general medical practice could and should play in PHC. This was because over $80 \%$ of the population visit a general practitioner (GP) at least once a year and this was usually their first point of contact with the health care system. 
Shortly after the Declaration of Alma-Ata, the WHO, supported by national and international nursing bodies, proposed that nurses would be the driving force behind the HFA movement as active partners in inter-professional teams, leaders in health care and resources to people rather than resources to other health professionals. Interestingly, at this time the government and the nursing profession acknowledged community health nurses as key players in PHC in Australia, but practice nurses (nurses who are employed in general medical practices) were not identified within this group. They seemed to be 'invisible' in the rhetoric of PHC.

However, in the 2001-2002 Commonwealth budget, \$104.3 million dollars was allocated to encourage GPs in areas (particularly rural and remote) of high workforce pressure to employ practice nurse (PNs) and to expand their role. The proposed role and function of the PN in Australia was then discussed at a national workshop in July 2001 where the participants articulated that the PN role should be complementary to that of the GP. Participants also noted that, as the role of the PN developed, it should include such aspects as: health promotion and education by promoting patient, carer and community well-being; sustaining general practice by contributing to better management of human and material resources; and, contributing to, and enhancing, the management and prevention of ill health (Department of Health and Ageing and Royal College of Nursing Australia, 2002). These suggested activities are fundamental to the goals of primary health care. In 2003, a further \$102 million was allocated for the employment of PNs in underserved urban areas and in the Medicare Plus package, there was also the generation of a Medical Benefit Scheme item for PN work involving immunisation and wound management. 
In the UK, the 1990 GP Contract resulted in GPs receiving financial remuneration for evidence of health promotion and primary disease prevention activities such as immunisation, cervical smears, registration health checks and annual checks for elderly people (Jeffreys, Clark \& Koperski, 1995). Many GPs chose to employ nurses to undertake this work because it was financially more profitable to do so than to undertake the extra work themselves. This was noted in a national census of PNs in England and Wales conducted in 1992 which demonstrated that the role of the PN had evolved from that of undertaking traditional, medically prescribed nursing tasks in a treatment room, to undertaking screening activities and health assessments of new patients with over $50 \%$ of the PNs managing chronic disease clinics (Atkin, Hirst, Lunt \& Parker, 1994). A more recent publication indicates that PNs in the UK also provide walk-in primary care services (Wilson, Pearson \& Hassey, 2002).

While limited research had been carried out on the current role of PNs in Australia (Linn 1969, 1979; Linn, Taylor \& Oborn, 1985; Linn, Taylor, Linn \& Johnston, 1990; DuntTemple-Smith \& Johnson. 1991; LeSueur \& Barnard 1993; Anastasiou, 1993; Keyzer, Hall, Mahnken \& Keyzer, 1996; Bonawit \& Watson 1996; Patterson, Del Mar \& Najman, 1999a, 1999b, 2000; Condon, Willis \& Litt, 2000), there is still debate in nursing and medical circles about what the focus of the role should be. For example, Lauder, Sharkey and Reel (2003) suggest that nurses and GPs in rural and remote areas should be interchangeable and that the focus should be on the competency of the person delivering care rather than the right of one discipline (medicine) to perform a particular role. In contrast, Campbell (1997) believes that public acceptance of nurses as GP substitutes would be poor, and therefore suggests that a better role for nurses in primary care would be health education and illness 
prevention. Three recently completed studies further explored the current and potential role of PNs in Australia from the perspectives of GPs, PNs and consumers. These studies, reported next, provide additional data important to this debate.

\section{The Studies}

Following a mailed survey to principal GPs and PNs in one Division of General Practice in southeast Queensland (see Patterson et al., 1999a, 1999b \& 2000), a follow-up qualitative study was undertaken with five of the GPs and five of the PNs to further explore their perceptions of the PN role. The selection of participants for this study was guided by the need for understanding and clarification of some of the outcomes from the survey in the context from which they originated. Each of the participants was interviewed for a period of 45 to 90 minutes. The interviews were guided by a set of open broad-ranging questions; the aim being exploration of issues as close as possible to a conversational ideal. Each interview was audiotape recorded and transcribed.

Using a "mid-range accounting system” (Miles \& Huberman, 1994, p. 61) analysis involved differentiating and combining the data firstly into broad areas, then into domains and finally into categories. Sections of the text were grouped into two broad areas according to whether they described the actual work of practice nurses or factors that appeared to have influenced the contribution of nursing to general practice. The data relating to the work of PNs were then classified into role domains and labelled. After repeated readings of the transcripts preliminary categories emerged within each of the role domains. These domains and categories were revised and refined throughout the process of data collection and analysis; some broken down into smaller 
units, some combined into a more inclusive domain or category and some discarded, while new ones emerged. A similar process was employed to classify the second group of data.

Two other studies, that were independently undertaken, (Cheek et al., 2003 \& Hegney et al., 2004) explored the perceptions of consumers about nurses in general medical practices. Participants represented a variety of general practice consumers including: carers of young and elderly people; the aged; young people; Indigenous Australians; people with a mental illness; those who had experience of PNs and those who did not. The Cheek et al. (2003) study, later referred to as the National Consumer Study, involved 170 consumers from four Australia states (New South Wales, Victoria, Queensland, and South Australia) and the two territories (Australian Capital Territory and the Northern Territory). In contrast, the 106 participants in the Hegney et al. (2004) study, later referred to as the Queensland Consumer Study, all lived in Queensland. However, in both studies, consumers from metropolitan, rural and remote locations were involved ensuring perspectives from consumers who had varying levels of access to primary, secondary and tertiary levels of health care. Recruitment of participants for both studies included multiple strategies such as: employing market research recruiting agencies, utilising contacts and networks of the investigators (eg carers associations and chronic illness support groups), and leaving information brochures at individual general practices or Divisions of General Practice.

Both studies generated data from semi-structured focus group interviews that were audio taped and later transcribed. The data analyses for both studies included the following steps: 
- Each transcript was studied group-by-group, by sampling of groups, and whole of data collated to give a sense of the whole;

- Themes and categories were identified group-by-group, by sampling of groups, and whole of data collated;

- Recurrent patterns were identified group-by-group, by sampling of groups, and whole of data collated; and

- Summative themes and research findings were developed.

A discussion between the two groups of researchers at the Inaugural National Practice Nurse Conference in 2003 identified significant similarities in the findings of these studies.

\section{The Findings}

An important differentiation was identified during the follow-up qualitative study of GPs and PNs. Two sub-groups of PNs were revealed that had many commonalities, but also some significant differences; for example, in the mode of employment of PNs. The dominant mode was for nurses to be employed and paid by the principal GPs of private medical practices. The other, less common, mode was for nurses to be employed in government sponsored health clinics in which GPs practised. In the latter situation, the GPs made no financial contribution to the employment of the nurses.

The data generated from the GP and PN interviews suggested that overall the PNs' scope of practice was broad in that they dealt with patients across the lifespan, both for acute and chronic conditions and preventive, restorative and rehabilitative nursing interventions. Findings revealed information about PNs' involvement in primary, secondary and tertiary disease/illness prevention. Primary prevention activities 
included administering immunisations, giving general advice about smoking, diet, exercise and stress reduction and keeping the practice stocked with educational pamphlets and posters. Secondary disease prevention was less commonly mentioned but some PNs were involved in general screening activities. In relation to tertiary disease prevention, the PNs contributed to educating patients with diabetes, asthma and hypertension about measures to monitor and treat their condition and prevent exacerbations. However, when the PNs did engage in health education, it was not usually the primary intention of the nursing intervention but undertaken while carrying out a medically delegated task like a wound dressing, blood pressure check or lung function test.

The PNs' role was found to encompass clinical, educative, administrative and supportive components. In their clinical role, they were predominantly dependent on medical delegation or direction. Their educative function was planned or expected when directed towards other staff but largely opportunistic when directed towards patients. As administrators, they were involved in managing financial, material and human resources as well as maintaining records and complying with regulations and standards. Supporting and promoting the doctor(s) and the practice were functions explicitly identified in the data as being of primary importance to the PN role. The doctors articulated this role in the following ways. One GP said, "being a sort of a solo practitioner here it's nice to have the back up of a registered nurse" and "I haven't actually thought of a practice nurse acting autonomously but rather together with the doctor as a helper or assistant" (GP-1). Similarly another said, "a nurse helps you to get the patients through more quickly” (GP-2) and again another expressed it this way: "I just felt the practice would run better with a trained nurse, 
sort of to assist in a lot of the things that practitioners do" (GP-4). When describing the contribution of nurses to general practice, this GP said: “It's basically a four man practice at any one time and each of us has a set day for operating so they assist us with that”.

Likewise the nurses described the ways in which they supported doctors in their role. One PN explained that when her current practice was established, doctors were recruited from other practices, which required her to have to "accommodate their individual needs and wants" (PN-1). Another described how the nurses in her practice assisted the doctors. "Usually you have got everything set up and ready and you've probably already done a few things depending on what has happened so it saves them a lot of time and energy" (PN-3). When asked to describe her relationship with the doctors in the practice, another said that one of the doctors called her "the handmaiden" (PN-4). When this point was pursued, she clarified that this was said in jest, however, she believed that was essentially what her role was. Another PN said several times that her main function was “facilitating the doctors' clinics” (PN-5). Although she had the capacity to practice autonomously, she consistently described her main role as supportive of the doctors.

The data also revealed that there was diversity between the PNs in the extent to which they engaged in each of the role categories. For example, one nurse employed in a large medical centre with a six bed treatment/minor surgery room and resuscitation bay described her role as largely clinical and akin to an accident and emergency nurse in a hospital. Another said she spent the majority of her time undertaking 
administrative activities, while yet another said she spent about half her time doing delegated clinical tasks and the other half doing reception/administrative activities.

Two of the PNs actually described themselves as primary health care nurses rather than as practice nurses. Both of these nurses were employed in health centres that incorporated other health services as well as general medical services and catered for specific population groups. One health centre was set up for the staff and students of a tertiary education institution and the other for Aboriginal people. In both, individuals could choose to consult the nurse and/or the doctor and the nurses directed health promotion activities to the client population as a whole, as well as to individuals.

In the first of these health centres, the nurse's position statement included the function “provide primary health care for students and staff”. The nurse employed explained that this meant providing treatment (that was within her training and competence) for illness and injury and health teaching for individuals. She also said "I do a reasonable amount of health promotion on the campus. I try and highlight two to three issues a semester, like breast cancer day or smokefree day. I also write articles about topical health issues for [the student newspaper] each time it comes out” (PN-5).

The nurse employed in the Aboriginal health centre described herself as "very much a primary health care nurse" because she did everything from "a top to toe health assessment to making sure someone's kids have got a proper lunch for school” (PN2). When asked what she thought her most important function was, she said: “Getting to know people, families, really well; making a real difference to their overall wellbeing." This nurse had a particular interest in diabetes and, as well as running a 
clinic for diagnosed diabetics, she conducted diabetes awareness and prevention campaigns for the rest of the health centre's clientele. She also made home visits to check on the general wellbeing of elderly patients.

The other PNs, who were privately employed, were asked about their involvement in health promotion. One said that she had requested that a notice board be put up in the waiting area and each week she promoted a different health topic. For example one week might be about mosquito eradication and the next might be about asthma awareness. She said that she tried to coincide her promotions with national 'awareness' days or with current media health issues.

Another nurse said, "I think you do a lot more health promotion and counselling here than just looking after patients like in a hospital. It seems like I have to think a lot more about their whole life and how things affect their families” (PN-3). Similarly another said that if health promotion meant educating and counselling patients about their illnesses and ways to prevent exacerbations, then she did that daily. However, she added, “I probably wouldn't have a lot of time to do any other health promotion; it is really quite a busy practice here" (PN-4).

Some of the nurses spoke about their family focus. Those who were involved with ante or postnatal care talked about the emphasis they placed on the family's wellbeing rather than just the mother's and baby’s. Most of them agreed that practice nursing was more concerned with family health than was hospital nursing, where nurses tended to come into contact with individuals for short episodes of care. They explained that they built up a strong relationship with people who had been coming to 
the practice for a long time and knew all about their family and work issues. One said, “I've looked after some kids from when I immunised them to when they've come about their acne" and another said, "I've seen some women through childbirth to menopause”.

The GPs were not asked specifically about their nurses’ roles in health promotion. However two of them raised this aspect of the role themselves. One of them said, "Our nurses are particularly good at promoting health, probably far better than us. I've heard them advising people when they've been giving them vaccinations for their overseas trips about how to avoid diseases" (GP-4). The other talked about the practice's plans to extend the nurses' role and said, "We'd like them to help us get some health promotion clinics up and running. A lot of our patients could benefit from a group program about weight control or stress management (GP-2).

These data suggest that PNs are involved in first level or primary care of individuals (most to a very limited extent) and engage in some form of preventive health care. A few PNs have a family/community focus in addition to their individual focus of care. The information also reveals that PNs believe that they provide holistic care to a greater extent than do their hospital colleagues.

While consumers could not specifically link comments made about the role of the PN to a PHC focus, across all the consumer focus groups there was widespread acceptance of there being a role for nurses in general practice as long as they were not substitutes for GPs in diagnosis and prescribing: "You will always look for the doctor's diagnosis regardless of what the nurse says"; "I see the nurses being 
experienced in caring and follow up care where doctors are trained to diagnose" (National Consumer Study participants). The focus of consumers on the doctor prescribing aligns with the view of most consumers that a general practice is a place where sickness care is provided.

Interestingly, consumers also articulated a perception or expectation of PNs as holistic and family oriented: "So a doctor might just look at your blood pressure and the sound of a heart or the lungs...but a nurse looks at it from the whole family perspective and how you're dealing with things" (QLD Consumer Study participant). "It would be nice if there was some sort of appointment for the carer to have a wellness check with the nurse...looking at the family in a holistic way" (National Consumer Study participant).

In between a caring and supportive role, and their wish for nurses not to diagnose, there was a grey area in relation to consumers' understanding of the PN role. Often what the consumers believed to be a nurse's skill was based on their hospital experiences, which is the context in which they normally witness nursing practice. However, many expressed the view that PNs could and should provide health education to themselves and their families: "I guess nurses could be good for describing to asthma patients how to use the medication and signs to look for...young mothers with immunisations" (QLD Consumer Study participant).

Another consistent expectation was that GPs and PNs should present as a united team and that each should respect and complement the other: "They should be working 
towards the same thing, the shared care of the person" (National Consumer Study participant); "It's important that the practice nurse is part of the team; it's an equal playing field” (QLD Consumer Study participant).

In both consumer studies there was a diversity of opinion about who should be the first point of contact in the general practice environment; some consumers believed that PNs should undertake a triaging role and see all patients first, while others thought they should always be seen by the GP first then referred to the PN for followup care. Despite these differing opinions, consumers were unanimous in expecting that any expansion in PN numbers or their scope of practice should improve accessibility to primary health care and not increase costs to themselves.

\section{Discussion}

The findings from these three studies extend previously reported findings of Patterson et al. (1999a \& 1999b). They identified that nursing's contribution to health promotion, including preventive care, was limited. These findings are not unexpected considering the evidence that, despite GPs' recognition of its importance, there are deficiencies in preventive care in Australian general practice (Buetow, 1995; GPSRG, 1998; Oldenburg \& Owen, 1995; Steven, Thomas, Eckerman, Browning \& Dickens, 2000; Waters, Haby, Wake \& Salmon, 2000).

Engagement in health promotion was found to be opportunistic rather than planned and focussed on interventions to free individuals from medically defined diseases, the aim being compliance with therapeutic procedures and advice. The broader concept of health promotion, as documented in the Ottawa Charter for Health Promotion (WHO, 
Health and Welfare Canada \& Canadian Public Health Association, 1986) was not pronounced in the PNs' reported practice. The Ottawa Charter states that health promotion action should encompass five strategies: build healthy public policy, create supportive environments, strengthen community action, develop personal skills and reorient health services to provide a greater balance between disease prevention and curative services. The evidence suggests that only one of these strategies (develop personal skills) was being utilised by most participants but the publicly funded PNs were also endeavouring to create supportive environments for their patients. However, it must be acknowledged that these findings are not unique to Australian PNs, but have been noted in the UK context (Bradford \& Winn, 1993) and in nursing generally (Dunlop \& Jacobs, 1995; Plymat, 1998).

The reality of practice nursing, as constructed by the GP and PN study participants, does not exemplify the expressed aspirations of the WHO and the ICN for nurses in PHC. While PNs are undoubtedly resources to individuals and families, this is not described as their primary function. Their allegiance and principal raison d'être is to assist and support the doctor. While they do educate people in 'health matters', this is not usually a planned and active pursuit, but rather an opportunistic activity incidental to other delegated clinical tasks.

Interestingly, the nurses in this study who were employed in publicly funded practices presented a slightly different picture from that of their privately employed colleagues. They had the opportunity to practice autonomously and appeared to have a far more 'person and family centred' focus than did the other study participants. However, their 
autonomy was limited to the extent that they could not prescribe medications or order diagnostic tests but would refer these activities to the GPs in the practice.

According to the WHO (1994), in most countries nurses are the most important group of health workers in terms of numbers, closeness to people and their health problems, and understanding of community needs. In Australia, nursing is the largest health profession comprising about 65\% of the health workforce (AIHW, 2002). However, there are significant differences in the distribution of these nurses between States and Territories and between metropolitan and non-metropolitan areas. In rural and remote areas, nursing employment is well above that of other health professions (AIHW, 1999) and these nurses are certainly recognised as 'the most important group of health workers'. The more remote the area, the more likely it is that PHC will be delivered by nurses because of the scarcity or absence of GPs. However, in metropolitan noninstitutional settings where there are approximately 90 GPs per 100,000 population (AMWAC \& AIHW, 1998) compared to 37 PNs per 100,000 and 57 community health nurses per 100,000, nurses are not as significant in terms of numbers or closeness to people's health problems. In this context, the importance of PNs in terms of PHC appears to be in how well they support and assist doctors to understand an individual's health needs.

Findings from both the national and Queensland consumer studies indicate that consumers perceive the PN role in much the same way, as do GPs. That is, nurses are valuable members of the general practice team but subservient to the GP. Given that consumers have limited, if any, understanding of PHC, it is not surprising they do not articulate confidence in PNs acting autonomously but rather as complementary to 
GPs, perhaps undertaking initial assessment for triage purposes and providing ongoing management, education and support under the GPs' delegation. They would also like them to be family oriented and holistic in their practice; supporting their emotional and social needs in the context of their family lives.

Most consumers articulated a limited understanding of nursing in general practice; those who had experienced a PN tended to describe the role with which they were familiar. While they could identify particular activities PNs could perform, they found difficulty in conceptualising the overall role. They acknowledged that their perceptions about the scope of practice nursing were informed by their understanding of general practice as a system that they access for their ill health or injury rather than a system for preventive health care.

\section{Conclusion}

It would appear that, currently, the role PNs play in PHC in Australia is generally limited in scope and in influence. While PNs do contribute to first contact care of individuals and sometimes families, they do not play a part in population health. Like GPs, their activities are predominantly focused on individualised treatment, episodic care and specific problems rather than on disease prevention, health promotion, continuous and comprehensive care. Providing hope that this reality can be changed are contemporary pre-registration nursing programs and a practice nurse professional association that facilitate a move beyond clinical skills to the principles and practices of PHC as envisaged by the WHO, including how nurses need to place a greater emphasis on relating to consumers what PHC can offer to them and how the role of the PN in PHC can contribute to better health outcomes. 
Most consumers utilise general practice for diagnosis and treatment of illness and injury, therefore have a perception of GPs and PNs only fulfilling that function. If general practice is to broaden its scope beyond primary medical care to primary health care, then a significant education program to change the mind-set of consumers is required. Similarly, education of the general public and of PNs and GPs is essential to the acceptance of PNs beyond that of assistants to the GPs. 


\section{References}

Anastasiou, M. (1993). Primary health care nursing in general practice. In 4th Annual Primary Health Conference. Development \& Diversity: Urban Primary Health Care. 18-19 November, 1993. Sydney: University of Sydney, Cumberland College of Health Sciences.

Atkin, K. Hirst, M. Lunt, N. \& Parker, G. (1994). The role and perceived training needs of nurses employed in general practice: observations from a national census of practice nurses in England and Wales. Journal of Advanced Nursing, 20, 46-52

Australian Institute of Health and Welfare. (1999). Nursing labour force 1998. AIHW Cat. No. HWL-14. Canberra: Author.

Australian Institute of Health and Welfare. (2002). Australia's health 1998: The eighth biennial health report of the Australian Institute of Health and Welfare. Canberra: Author.

Australian Medical Workforce Advisory Committee \& Australian Institute of Health and Welfare. (1998). Medical workforce supply and demand in Australia: A discussion paper, AMWAC Report 1998.8, AIHW Cat. No. HWL 12. Sydney: AIHW. 
Bradford, M., \& Winn, S. (1993). Practice nursing and health promotion: A case study. In J. Wilson-Barnett, \& J. Macleod Clark (Eds.), Research in health promotion and nursing (pp. 119-131). London: Macmillan.

Bonawit, V., \& Watson, L. (1996). Nurses who work in general medical practices: A Victorian survey. Australian Journal of Advanced Nursing, 13 (4), 28-34.

Buetow, S. (1995). What do general practitioners and their patients want from general practice and are they receiving it? Social Science \& Medicine, 40 (2), 213-221.

Campbell, S. (1997).The newest gatekeepers: nurses take on the duties of primary care. Health Care Strategic Management, 15 (3), 14-15.

Cheek, J., Price, K., Mott, K., Wilkinson, D., Beilby, J. \& Dawson, A. (2003).

Consumer perceptions of nurses and nursing in general practice. Department of Health and Ageing. Completed 2002 published on-line 2003 http://www.health.gov.au/hsdd/gp/nursing/index.htm

Condon, E., Willis, E., \& Litt, J. (2000). The role of the practice nurse: An exploratory study. Australian Family Physician, 29 (3), 272-277.

Department of Health and Ageing and Royal College of Nursing, Australia. (2002).

Nursing in general practice - Fact Sheet 1. DHA, Canberra, Australia. 
Dunlop, M., \& Jacobs, J. (1995). Primary health and nursing: Strange bedfellows? In

G. Gray, \& R. Pratt (Eds.), Scholarship in the Discipline of Nursing (pp. 277-292).

South Melbourne: Churchill Livingstone.

Dunt, D. R., Temple-Smith, M. J., \& Johnson, K. A. (1991). Nursing outside hospitals: The working experience of community nurses. Job characteristics. International Journal of Nursing Studies, 28 (1), 27-37.

General Practice Strategy Review Group. (1998). General practice: Changing the future through partnerships. Report of the General Practice Strategy Review Group. Canberra: Commonwealth Department of Health and Family Services.

Hegney, D., Buikstra, E., Fallon, T., Martin-McDonald, K., Patterson, E. \& Rees, S. (2004). Consumer perceptions of practice nursing. Centre for Rural and Remote Area Health, University of Southern Queensland, Toowoomba, Queensland, Australia.

Jeffreys, L., Clark, A. \& Koperski, M. (1995). Practice nurses' workload and consultation patterns. British Journal of General Practice, 45, 415-418.

Keyzer, D. M., Hall, J., Mahnken, J., \& Keyzer, K. (1996). Gum Trees and Windmills: A study in the management of time, space and the self-concepts of community-based nurses in one rural area of Victoria, Australia. Warnambool, Victoria: Deakin University. 
Lauder, W., Sharkey, S. \& Reel, S. (2003). The development of family health nurses and family nurse practitioners in remote and rural Australia. Australian Family Physician, 32 (9), 750-752

Le Sueur, H., \& Barnard, A. (1993). Nurses in general practice in metropolitan Western Australia: A report to the Department of Health, Housing and Community Services 92/06341 [D92/0723]. Canberra: Department of Health, Housing and Community Services.

Linn, J. (1969). The health team in general practice: Summary of a survey in rural practice. Annals of General Practice, 14 (162), 162-168.

Linn, J. (1979). Chronic illness in the elderly - the role of the nurse. (Research Report Series, Monograph No. 4. ed.). Canberra, A.C.T.: Department of Health.

Linn, J., Taylor, W. \& Oborn, J. (1985). The trained nurse in general practice. Medical Journal of Australia, 142 (1), 28-29.

Linn, J.T, Taylor, W., Linn, J.E., \& Johnston, A. (1990). Care of the elderly in general practice: The nurse's role. The Medical Journal of Australia, 152 (12), 676. 
Miles, M. B., \& Huberman, A.M. (1994). Qualitative data analysis: An expanded sourcebook. Thousand Oaks: Sage.

National Health Strategy. (1991). The Australian health jigsaw: Integration of health care delivery. Issues paper No 1. Canberra: Department of Health, Housing and Community Services.

National Health Strategy. (1992). The future of general practice: Issues paper No. 3. Canberra: Department of Health, Housing and Community Services.

Oldenburg, B., \& Owen, N. (1995). Preventive care in general practice in Australia: A public health perspective. Patient Education and Counselling, 25, 305-310.

Patterson, E., Del Mar, C., \& Najman, J. (1999a). A descriptive study of nurses employed by general practitioners in south-east Queensland. Australian Journal of Advanced Nursing, 17 (2), 13-20.

Patterson, E., Del Mar, C., \& Najman, J. (1999b). Nursing's contribution to general practice: General practitioners' and practice nurses' views. Collegian, 6 (4), 33-39. 
Patterson, E., Del Mar, C., \& Najman, J. (2000). Medical receptionists in general practice: Who needs a nurse? International Journal of Nursing Practice, 6, 229-236.

Plymat, K. R. (1998). Health promotion in nursing practice: Discussion paper No. 1. ACT, Australia: Royal College of Nursing Australia.

Steven, I., Thomas, S., Eckerman, E., Browning, C., \& Dickens, E. (2000). The provision of preventive care by general practitioners measured by patient completed questionnaires. Journal of Quality in Clinical Practice, 19 (4), 195-201.

Waters, E. B., Haby, M. M., Wake, M., \& Salmon, L. A. (2000). Public health and preventive healthcare in children: Current practices of Victorian GPs and barriers to participation. Medical Journal of Australia, 173 (2), 68-71.

Wilson, A., Pearson, D. \& Hassey, A. (2002). Barriers to developing the nurse practitioner role in primary care: The GP perspective. Family Practitioner, 19 (6), 641-646.

World Health Organization. (1978). Primary health care. Report of the international conference on primary health care. Alma Ata, USSR, 6-12 September. Heath for All Series No 1. Geneva: Author. 
World Health Organization, Health and Welfare Canada, \& Canadian Public Health Association. (1986). Ottawa Charter for Health Promotion. 1st International Conference on Health Promotion , November 7-21, Ottawa, Canada. Geneva: Authors.

World Health Organization. (1994). Nursing beyond the year 2000: Report of a WHO study group. WHO Technical Report Series, No 860. Geneva: Author. 